\title{
Current Status of Nursing Research on Beckwith Wiedemann Syndrome Based on Co-word Analysis
}

\author{
Email address: \\ 41867582@qq.com (Lilan He) \\ ${ }^{*}$ Corresponding author
}

Yun Chen, Meng Zhang, Lilan He*

Department of Neonatology, The First Affiliated Hospital of Jinan University, Guangzhou, China

\section{To cite this article:}

Yun Chen, Meng Zhang, Lilan He. Current Status of Nursing Research on Beckwith Wiedemann Syndrome Based on Co-word Analysis. World Journal of Public Health. Vol. 4, No. 4, 2019, pp. 102-108. doi: 10.11648/j.wjph.20190404.15

Received: November 7, 2019; Accepted: November 28, 2019; Published: December 11, 2019

\begin{abstract}
Using the "Beckwith Wiedemann" "care" or "Beckwith Wiedemann" "nursing" literature in the core collection of Web of Science (WOS) as a data source to understand and analyze the current status of nursing research in Beckwith Wiedemann syndrome for Beckwith Wiedemann syndrome care Provide clinical and research references. The selected literature titles are imported into the literature bibliographic information statistical analysis tool (SATI) to perform frequency statistics on the subject words, matrix is generated, and the results are imported into SPSS 22.0 for drawing cluster analysis and multidimensional scale analysis map. Based on the clustering analysis of high-frequency subject words and the results of multidimensional scaling analysis, the research focus on Beckwith Wiedemann syndrome nursing is: Beckwith Wiedemann syndrome nursing research has experienced a slow development period, and is now in a prosperous period, but the overall number of studies is low. The content coverage is comprehensive but not in-depth and should be improved. The research and exploration of Beckwith Wiedemann syndrome care can be further combined with the current research hotspots to improve the quantity and quality of Beckwith Wiedemann syndrome nursing research.
\end{abstract}

Keywords: Co-word Analysis, Beckwith Wiedemann Syndrome, Research Status, Nursing

\section{Introduction}

Beckwith Wiedemann Syndrome (BWS) is an overgrowth syndrome that causes all or part of the body to grow larger than normal. BWS overgrowth and overgrowth (large birth weight, height, head circumference), giant tongue (large tongue), half body asymmetry (one side of the body grows wholly or partially larger than the other side) and some Specific types of tumors are associated with other physiological characteristics [1]. This syndrome was first described by Dr. Beckwith in 1963 and Dr. Wiedemann in 1964 [2]. The incidence of BWS in newborns is approximately 1: 14,500. Although BWS can be inherited (autosomal dominant), most cases are still considered to be new or sporadic genetic mutations. The extent to which each patient is affected is very different [3]. Because BWS is often accompanied by diseases such as giant tongue, enlarged viscera, and hypertrophy of the half, the care of BWS is especially important for patients [4]. With the development of medicine, Beckwith Wiedemann's treatment has greatly improved, and Beckwith Wiedemann's care has entered a new stage of development. The co-word analysis method reflects the intensity of the association between text keywords by frequency ordering in the common word matrix table. Cluster analysis is based on the numerical characteristics of things to calculate the relationship between each variable or sample [5]. Multidimensional scale analysis method is to input the similarity degree matrix and find the coordinates, so as to judge the degree of similarity between the stimuli according to the length of the distance. Therefore, this study intends to explore the research situation and hotspots in the field of Beckwith Wiedemann syndrome nursing from the perspective of bibliometric analysis, based on the theory of co-word analysis, using cluster analysis and multi-dimensional scale analysis, for Beckwith Wiedemann syndrome. Nursing provides reference and reference. 


\section{Research Design}

\subsection{Data Source}

The data source for this study is the core collection of Web of Science. The search strategy is: subject $=($ "Beckwith Wiedemann" and "care") or ("Beckwith Wiedemann" and "nursing"), time span = "1900-2019", language is limited to "English", September 2019 A total of 65 articles were retrieved on the 24th; through the removal of duplicates and screenings, a total of 54 records of Beckwith Wiedemann's nursing field with the theme of "Beckwith Wiedemann" and "care" or "Beckwith Wiedemann" and "nursing" were obtained. All search records and referenced references, the file format is plain text.

\subsection{Research Methods}

This study mainly uses the method of co-word analysis and cluster analysis. Co-word analysis is a kind of content analysis method [6]. The main principle is that hot words with high frequency appearing in the literature database of a certain research field can express the research hot topics and development trends in the field; the keywords are co-occurring in the same [7]. The more times the keywords co-appear in the same literature, the closer the relationship between the two topics they express. By clustering the hotspot keyword co-occurrence matrix, multiple closely related topics can be clustered into relatively independent conceptual clusters, indicating hotspots in a certain research field, in which the same cluster has the most similar topicality. The similarity between clusters and clusters is minimal [8]. This paper obtains the hotspot keywords in the field of library and information research driven by big data through SATI3.2, and generates hotspot keyword co-occurrence matrix for co-word analysis [9]. Then use SPSS20.0 for cluster analysis and combing big data [10]. Driven by the research hot topics in the field of library and information, in order to provide reference for library and information research under the background of big data.

\section{Research Results}

\subsection{Overview of Field Research}

\subsubsection{Annual Distribution Volume Distribution Trend}

The annual distribution of the volume of publications is the mapping of the amount of literature on the time nodes, and it is also the specific performance of the research heat over time. The year data in the valid topics collected is entered into Excel and arranged in ascending order, as shown in Figure 1.

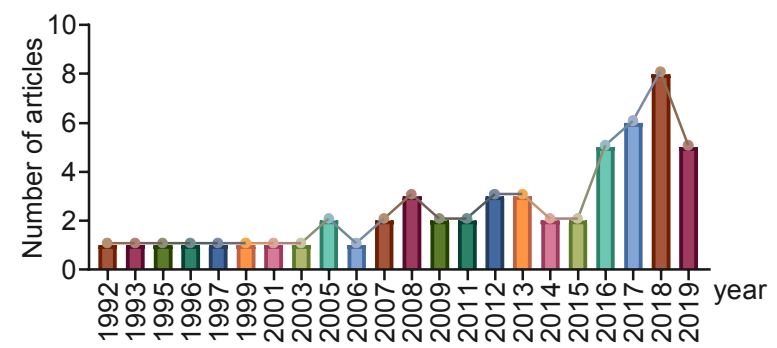

Figure 1. The distribution of the volume of publications with "Beckwith Wiedemann" "care" or "Beckwith Wiedemann" "nursing" as the theme.

It can be seen from Figure 1 that the overall development of Beckwith Wiedemann syndrome nursing research can be divided into two periods: from 1992 to 2007, it is the germination period, and from 2008 to the present, it is the exploration period. The distribution characteristics of the literature in each period are as follows: In the germination period with a span of 16 years, the annual literature volume is less than 2. The earliest paper "Anesthetic considerations of an infant with Beckwith-Wiedemann syndrome" was published by Tobias JD of Vanderbilt University in the United States in 1992 in J Clin Anesth. In the 12-year exploratory period, the annual literature volume has remained basically between 2 and 10, of which 2016, 2017, 2018, and 2019 have the largest number of publications and are increasing year by year, accounting for $64.8 \%$ of the total literature for four years, indicating the world. The study of Beckwith Wiedemann Syndrome Nursing is in the process of exploration, but the total research is extremely low, and more researchers should be involved in the study of Beckwith Wiedemann syndrome care.

\subsubsection{Research Power Country (Region) Distribution}

The distribution of research power countries (regions) can reflect the spatial distribution of the main research forces in a research field. According to the statistical analysis of 54 literatures, the Beckwith Wiedemann syndrome nursing research force is distributed in 250 countries in 35 countries. In research institutions. Table 1 shows the top 20research institutions and the top 25 research institutions of research power.

Table 1. The top 20 research institutions and the top 20 research institutions of research power.

\begin{tabular}{lllll}
\hline Countries & Numbers & Percentage & Institutions & Numbers \\
\hline USA & 23 & $42.59 \%$ & CNR & 5 \\
ITALY & 12 & $22.22 \%$ & UNIV PENN & 5 \\
ENGLAND & 9 & $16.67 \%$ & IST AUXOL ITALIANO & $9.26 \%$ \\
FRANCE & 9 & $16.67 \%$ & UNIV MILAN & 4 \\
GERMANY & 9 & $16.67 \%$ & UNIV NAPLES 26 & 4 \\
DENMARK & 6 & $11.11 \%$ & UNIV PARIS 05 & 4 \\
NETHERLANDS & 5 & $9.26 \%$ & UNIV SOUTHAMPTON & 4 \\
SPAIN & 5 & $9.26 \%$ & CHILDRENS HOSP & 4 \\
AUSTRALIA & 3 & INSERM & $7.41 \%$ & 3 \\
BELGIUM & 3 & $5.56 \%$ & RHEIN WESTFAL TH AACHEN & 3 \\
\hline
\end{tabular}




\begin{tabular}{lllll}
\hline Countries & Numbers & Percentage & Institutions & Pumbers \\
\hline CANADA & 3 & $5.56 \%$ & UNIV HELSINKI & 3 \\
FINLAND & 3 & $5.56 \%$ & UNIV TORINO & 3 \\
JAPAN & 3 & $5.56 \%$ & UNIV TURIN & 3 \\
SWEDEN & 3 & $5.56 \%$ & ACAD MED CTR & $5.56 \%$ \\
BRAZIL & 2 & $3.70 \%$ & AUSTRALIAN NATL UNIV & 2 \\
CYPRUS & 2 & $3.70 \%$ & CHILDRENS MEM HLTH INST & 2 \\
MALTA & 2 & $3.70 \%$ & CYPRUS INST NEUROL GENET & 2 \\
POLAND & 2 & HANNOVER MED SCH & $3.70 \%$ & 2 \\
TAIWAN & 2 & $3.70 \%$ & HELSINKI UNIV HOSP & $3.70 \%$ \\
TURKEY & 2 & $3.70 \%$ & HOP COCHIN & 2 \\
\hline
\end{tabular}

From the statistical results, the following characteristics can be found: (1) There are 8 countries (regions) with a volume of more than 5, accounting for $14.2 \%$, and about $99.4 \%$ of the research institutions contribute 2 or less documents, and the number of publications is more than 2 There are only 13 institutions, accounting for $0.52 \%$. (2) From the frequency of writing, the United States has the largest contribution, and the number of publications is the second in Italy; there are 2 countries (regions) with more than 10 articles, and the number of publications is more than 10 There are 0 research institutes, ranking first in the Italian CNR research institute with 5 papers and the University of Pennsylvania in the United States. Only one of the top seven research institutions in the publications is not an American research institution, indicating that the United States has a leading edge in eckwith Wiedemann syndrome care research.

\subsubsection{Core Author Analysis and Main Publications}

54 articles from 410 authors and 46 academic journals are shown in Table 2. The author with the largest number of publications is Professor RICCIO A, who published a total of six articles, all of which are first authors. The authors who posted more than 2 articles were 12, accounting for $0.03 \%$. Four authors with more than 4 articles were published. After importing the bibliographic data into Hist Cite software, Total Local Citation Score was found. The highest author of TLCS was MUSSA A, up to 7 times. A statistical analysis of 46 journals found that the journals with the largest number of articles were JOURNAL OF PEDIATRIC SURGERY and NATURE REVIEWS ENDOCRINOLOGY, with 3 articles respectively, accounting for $11.11 \%$ of the total. From the perspective of TLCS, the most cited journal is ENDOCRINE REVIEWS.

Table 2. Top 20 research authors and top 20 published journals.

\begin{tabular}{|c|c|c|c|c|c|}
\hline Author & Numbers & Percentage & Publications & Numbers & Percentage \\
\hline RICCIO A & 6 & $11.11 \%$ & JOURNAL OF PEDIATRIC SURGERY & 3 & $5.56 \%$ \\
\hline FERRERO GB & 5 & $9.26 \%$ & NATURE REVIEWS ENDOCRINOLOGY & 3 & $5.56 \%$ \\
\hline MUSSAA & 5 & $9.26 \%$ & AMERICAN JOURNAL OF MEDICAL GENETICS PART A & 2 & $3.70 \%$ \\
\hline RUSSO S & 5 & $9.26 \%$ & CURRENT OPINION IN PEDIATRICS & 2 & $3.70 \%$ \\
\hline EGGERMANN T & 4 & $7.41 \%$ & EUROPEAN JOURNAL OF HUMAN GENETICS & 2 & $3.70 \%$ \\
\hline GRONSKOV K & 4 & $7.41 \%$ & MEDICAL AND PEDIATRIC ONCOLOGY & 2 & $3.70 \%$ \\
\hline TUMER Z & 4 & $7.41 \%$ & MOLECULAR GENETICS AND METABOLISM & 2 & $3.70 \%$ \\
\hline MACKAY DJG & 3 & $5.56 \%$ & ADVANCES IN EXPERIMENTAL MEDICINE AND BIOLOGY & 1 & $1.85 \%$ \\
\hline MONK D & 3 & $5.56 \%$ & ALCOHOL AND CANCER & 1 & $1.85 \%$ \\
\hline NETCHINE I & 3 & $5.56 \%$ & ANNALS OF ONCOLOGY & 1 & $1.85 \%$ \\
\hline TEMPLE IK & 3 & $5.56 \%$ & ANTICANCER RESEARCH & 1 & $1.85 \%$ \\
\hline AIGRAIN Y & 2 & $3.70 \%$ & ARCHIVES DE PEDIATRIE & 1 & $1.85 \%$ \\
\hline ARNOUX JB & 2 & $3.70 \%$ & ARCHIVOS ARGENTINOS DE PEDIATRIA & 1 & $1.85 \%$ \\
\hline BAUER S & 2 & $3.70 \%$ & $\begin{array}{l}\text { BEST PRACTICE RESEARCH CLINICAL OBSTETRICS } \\
\text { GYNAECOLOGY }\end{array}$ & 1 & $1.85 \%$ \\
\hline BOONEN SE & 2 & $3.70 \%$ & CLEFT PALATE CRANIOFACIAL JOURNAL & 1 & $1.85 \%$ \\
\hline BRIOUDE F & 2 & $3.70 \%$ & CLINICAL EPIGENETICS & 1 & $1.85 \%$ \\
\hline COCCHI G & 2 & $3.70 \%$ & CLINICAL GENETICS & 1 & $1.85 \%$ \\
\hline DE LONLAY P & 2 & $3.70 \%$ & CLINICAL OBSTETRICS AND GYNECOLOGY & 1 & $1.85 \%$ \\
\hline
\end{tabular}

\subsection{Cluster Analysis of Keywords}

\subsubsection{Generation of Hot Keyword List}

The generation of hot keyword list authors imported the above 54 titles into the statistical analysis tool SATI3.2 of the bibliographic information developed by Liu Qiyuan. After de-re-transforming and extracting keywords, a total of 190 keywords were obtained (Figure 2), which have similar meanings. Or the words with no practical meaning are combined and removed, and arranged in descending order of frequency to obtain 19 keywords whose frequency is greater than or equal to 2, and generate a list of hotspot keywords as shown in Table 3. As can be seen from Table 3, a series of keywords have been generated around the field of Beckwith Wiedemann syndrome care, such as Beckwith-Wiedemann syndrome, hepatoblastoma, placenta, and cancer predisposition. 


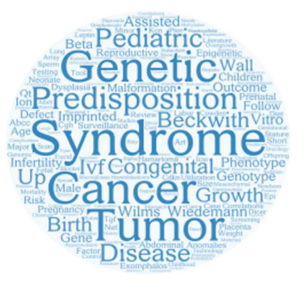

Figure 2. Cloud map of hot keywords.

Table 3. Keywords with frequency greater than or equal to 2.

\begin{tabular}{ll}
\hline Keywords & frequency \\
\hline Beckwith-Wiedemann syndrome & 9 \\
hepatoblastoma & 3 \\
placenta & 3 \\
cancer predisposition & 3 \\
Beckwith-Wiedemann & 3 \\
overgrowth & 3 \\
congenital hyperinsulinism & 2 \\
screening & 2 \\
cancer genetics & 2 \\
assisted reproductive technology & 2 \\
Omphalocele & 2 \\
Genetic testing & 2 \\
Outcomes & 2 \\
assisted reproduction & 2 \\
birth weight & 2 \\
cancer & 2 \\
Mortality & 2 \\
predisposition & 2 \\
pancreatectomy & 2 \\
\hline
\end{tabular}

\subsubsection{Generation of Hotspot Keyword Dissimilar Matrices}

In order to further understand the relationship between hotkey keywords, grasp the research status of the library and information field driven by big data, pair the 19 hot keywords obtained above into two pairs, pre-process, and count every two keywords together. The frequency of occurrence, the $19 * 19$ hotspot keyword co-occurrence matrix is constructed. Since the word frequency cannot reflect the true connection of each keyword, it is necessary to construct a similarity matrix to eliminate the impact of frequency disparity on the result. In this paper, the Equivalence coefficient is used to convert the multivalued co-occurrence matrix into the $[0,1]$ interval. The similarity matrix calculation method is to divide the square of the frequency of the two keywords in the co-occurrence matrix by the frequency of occurrence of the two keywords. The product is as shown in formula (1): E = FABFA * FB2 (1) where $\mathrm{E}$ represents the value of the Equivalence coefficient, $\mathrm{FAB}$ represents the frequency at which the keywords $\mathrm{A}$ and $\mathrm{B}$ co-occur in the literature, and FA represents the keyword A in the literature. The frequency of occurrence of concentration, FB indicates the frequency of occurrence of keyword $B$ in the literature set; the larger the value of $\mathrm{E}$, the stronger the degree of association between the two keywords. Because the value of 0 in the similar matrix is too large, the error is easily caused by excessive statistic. Therefore, each value in the similar matrix is subtracted from 1 to obtain the dissimilar matrix of the hotspot keywords. See Table 4 (limited to the space, only listed) The partial value of the matrix is opposite to the similarity matrix. The larger the value in the dissimilar matrix, the smaller the distance between the two keywords, the smaller the correlation is. On the contrary, the smaller the value, the distance between the keywords. The closer, the greater the correlation.

Table 4. 19 hot keywords dissimilarity matrix.

\begin{tabular}{|c|c|c|c|c|c|c|c|c|c|c|}
\hline & $\mathbf{A}$ & B & C & D & $\mathbf{E}$ & $\mathbf{F}$ & G & $\mathbf{H}$ & I & $\mathbf{S}$ \\
\hline $\mathrm{A}$ & 0 & 0.963 & 0.9444 & 0.963 & 1 & 0.963 & 0.9444 & 0.9444 & 0.9444 & 0.9444 \\
\hline B & 0.963 & 0 & 1 & 1 & 1 & 1 & 1 & 0.8333 & 1 & 1 \\
\hline $\mathrm{C}$ & 0.9444 & 1 & 0 & 1 & 1 & 1 & 1 & 1 & 1 & 1 \\
\hline $\mathrm{D}$ & 0.963 & 1 & 1 & 0 & 0.8889 & 0.5556 & 1 & 1 & 1 & 1 \\
\hline $\mathrm{E}$ & 1 & 1 & 1 & 0.8889 & 0 & 0.8889 & 1 & 1 & 1 & 1 \\
\hline $\mathrm{F}$ & 0.963 & 1 & 1 & 0.5556 & 0.8889 & 0 & 1 & 1 & 1 & 1 \\
\hline $\mathrm{G}$ & 0.9444 & 1 & 1 & 1 & 1 & 1 & 0 & 1 & 1 & 0 \\
\hline $\mathrm{H}$ & 0.9444 & 0.8333 & 1 & 1 & 1 & 1 & 1 & 0 & 1 & 1 \\
\hline I & 0.9444 & 1 & 1 & 1 & 1 & 1 & 1 & 1 & 0 & 1 \\
\hline $\mathrm{J}$ & 1 & 1 & 1 & 1 & 1 & 1 & 1 & 1 & 1 & 1 \\
\hline K & 1 & 1 & 1 & 1 & 1 & 1 & 1 & 1 & 1 & 1 \\
\hline $\mathrm{L}$ & 1 & 1 & 1 & 0.8333 & 1 & 1 & 1 & 1 & 1 & 1 \\
\hline M & 1 & 1 & 1 & 1 & 1 & 1 & 1 & 1 & 1 & 1 \\
\hline $\mathrm{N}$ & 1 & 1 & 1 & 1 & 1 & 1 & 1 & 1 & 1 & 1 \\
\hline $\mathrm{O}$ & 1 & 0.8333 & 1 & 1 & 1 & 1 & 1 & 1 & 1 & 1 \\
\hline $\mathrm{P}$ & 1 & 1 & 1 & 1 & 1 & 1 & 1 & 1 & 1 & 1 \\
\hline Q & 1 & 1 & 1 & 1 & 1 & 1 & 1 & 1 & 1 & 1 \\
\hline $\mathrm{R}$ & 1 & 1 & 1 & 1 & 1 & 0.8333 & 1 & 1 & 1 & 1 \\
\hline S & 0.9444 & 1 & 1 & 1 & 1 & 1 & 0 & 1 & 1 & 0 \\
\hline
\end{tabular}

A: Beckwith-Wiedemann syndrome B: hepatoblastoma C: placenta D: cancer predisposition E: Beckwith-Wiedemann F: overgrowth G: congenital hyperinsulinism H: screening I: cancer genetics J: assisted reproductive technology K: Omphalocele L:Genetic testing M:Outcomes N: assisted reproduction O: birth weight P: cancer Q: Mortality R: predisposition S: pancreatectomy

\subsection{Hot Keyword Clustering}

Cluster analysis can classify keywords into different classes or clusters. Relevant keywords will be aggregated into the same class or cluster, and there will be greater differences between different classes or clusters. Introduce the dissimilar matrix into SPSS statistical analysis software, select "system clustering analysis" and "connection between groups", and 
then generate a tree cluster diagram of research hotspot keywords in the field of library and information driven by big data. The system clustering analysis tree diagram can visually display the specific situation of the clustering process and the combination of keywords in each clustering process. As shown in Figure 3, Beckwith Wiedemann syndrome care focuses on five aspects: Beckwith-Wiedemann syndrome and hepatoblastoma, Beckwith Wiedemann and genetic variation, assisted reproduction, hyperinsulinemia and pancreatic resection, cancer and death.

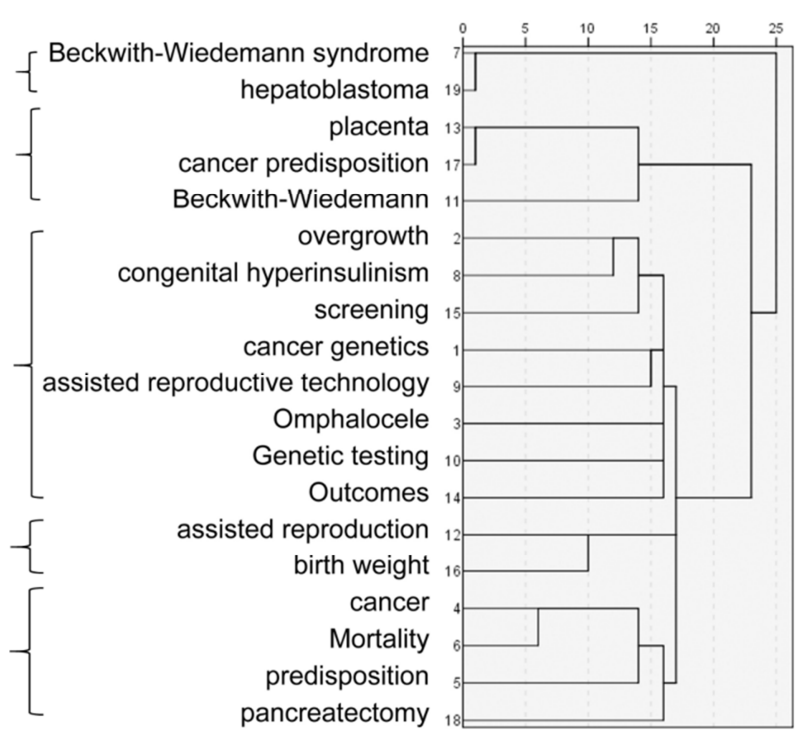

Figure 3. Clustering results of 19 hot words.

\section{Discussion}

BWS is a group of clinical syndromes characterized by three major features: giant tongue, umbilical bulge and overgrowth [11]. Other features include visceral enlargement, unilateral hypertrophy, linear depression of the earlobe, adrenocortical cell enlargement, and hypoglycemia after birth. The incidence of BWS is about $1 / 13700$, the distribution is $85 \%$, and the family inheritance is $15 \%$ [12]. Familial inheritance is unilateral inheritance of the maternal line, indicating that its pathogenesis is associated with genomic imprinting. The onset of BWS may be the result of multiple genes. Recent studies have shown that about $75-80 \%$ of the pathogenic genes of BWS are located in the $11 \mathrm{p} 15.5$ region, and other pathogenicity-related loci may be located on chromosomes 1941, 642. Loss or base methylation results in the onset of an individual. BWS diagnosis lacks specific indicators and is more likely to be diagnosed after delivery [13]. There are no guidelines for prenatal diagnostic criteria. Some scholars suggest that there are 2 main criteria (abdominal wall defect, giant tongue, giant body) or a major standard plus 2 secondary criteria (mega kidney or kidney malformation, adrenal hypertrophy, genetic or chromosomal abnormalities, polyhydramnios, etc.) can be diagnosed, but more case support is needed to reach an agreement [14]. BWS is difficult to detect early in the birth examination and is easily missed. The reasons are as follows: 1. BWS is a rare disease, and obstetrics and imaging doctors lack sufficient knowledge of the disease. 2. BWS individuals lack characteristic characteristics in early pregnancy, and accelerate the growth in the middle and late pregnancy. Only the abdominal organs and tongues are enlarged, and the images can be found. 3. Missed cases are mainly characterized by minor deformities and defects. 4. Pregnant women are not strictly in accordance with prenatal screening guidelines. 5 . The cause is complicated and it is difficult to re-evaluate the risk. Domestic molecular genetic testing is limited by technology.

The cause is related to the genomic imprint. According to Mendel's law of inheritance, the two alleles from the father and the mother are expressed equidistantly. If only the parent or mother gene is selectively expressed, the gene is used as a mark to control its gene [15]. The expression study found that the $11 \mathrm{p} 15.5$ region of BWS is an imprinted gene cluster, its p57 mutation, IGF2/H19 transcriptional abnormality chromosomal translocation (inversion) and methylation of CpG island upstream of LITI gene may be the cause of BWS. These genes have a tendency to share regulatory mechanisms, so the onset of BWS may be the result of multiple genes, but the exact pathogenesis remains to be further studied [16].

Pathological manifestations in addition to the obvious hypertrophy of the lingual muscle fibers, myocardial liver and kidney adrenal pancreas and other visceral disproportionate hypertrophy domestic report 1 case of neonatal BWS autopsy pathology: two enlarged kidney volume accounted for $2 / 3$ of the abdominal cavity, kidney Most of the small balls mature, the size of the tubular expansion is different, the number of islet cells increases, and the volume of insulin increases significantly, which may be accompanied by an increase in growth hormone levels [17]. The incidence of BWS hypoglycemia is about $50 \%$. A series of studies on BWS hypoglycemia suggest that about $80 \%$ of cases are mild and asymptomatic, requiring only oral or intravenous glucose, and the other $20 \%$ are persistent (duration over) 1 week), and it is difficult to control hypoglycemia to 3 years after birth, and hyperinsulinemia associated with mental impairment is the cause of transient and persistent hypoglycemia in BWS. Many case reports provide BWS metabolic defects [18]. The data, but their uniformity is small, the possible reason is due to the different focus of the different authors' surveys, but one thing is more consistent, that is, the level of blood sugar and the reactivity of insulin to beta cell secretagogues due to the data [19]. Limitations, the relationship between beta cell abnormalities and hyperinsulinemia remains unclear, but all patients with BWS hypoglycemia are characterized by hyperinsulinemia and inappropriate secretion of insulin during hypoglycemia; excessive sustained insulin response and/or Glucose-induced hypoglycemia is often reported when glucagon is detected at low and high blood glucose levels, and persistent hyperinsulinemia leads to a decrease in blood glucose concentration [20]. Tolbutamide treatment can induce Rapid and long-lasting high insulin response, glucose concentration decreased serum arginine and leucine effect on $\beta$ cells are normal [21].

Since there is currently no established genetic testing method for BWS, the diagnosis of BWS can and should be based on 
clinical diagnosis rather than blood testing. The diagnosis of BWS is based on clinical suspicion and exhibits two of the top 5 symptoms, and other minor symptoms are also helpful in diagnosis [22]. Unsupervised hypoglycemia, preterm birth (and associated risks) and the likelihood of developing a tumor are the greatest risks for BWS children. Usually hypoglycemia is temporary, but in some cases it will continue into early childhood and in severe cases may require surgery (removing some or all of the pancreas) [23]. Any suspicious hypoglycemia after birth requires immediate examination and treatment. Although there may be problems associated with preterm birth, medical science has been able to help more premature babies and their future is brighter than it was a few years ago. Abdominal cancer is the biggest risk for BWS children [24]. Approximately $5-10 \%$ of BWS children develop cancer. The most common tumors associated with BWS are: Wilm's tumor of the kidney, tumor of the liver (hepatoblastoma), and relatively rare adrenal and neuroblastoma. Although there is still a lack of understanding of the cancer risk for each particular individual, it appears that those with half-limb limb hypertrophy or hyperplasia are at greater risk for developing a tumor than those without these symptoms [25].

Children with BWS generally have small mandibular joints and have difficulty eating. In order for children to breathe normally, they have to enlarge the oropharynx, that is, to expand the oropharynx or reduce the contents of the oral cavity [26]. We combine the experience of treating small mandibular deformities and take the extended jaw. The method (forward and downward) increases the anteroposterior diameter and the up and down diameter of the oral cavity, thereby increasing the true oral cavity; on the other hand, reducing the tongue to reduce the space occupied by the mouth, thereby relatively increasing the volume of the oral cavity, thus Feeding with a spoon or teat can solve the problem of eating difficulties [27]. Currently reported Beckwith-Wiedemann syndrome, the method used is to treat the giant tongue, such as Mehmet and other lasers to remove the huge tongue, but the treatment of other malformations is less involved, only Achara and other treatment of the giant tongue after the treatment of the half face hypoplasia. To prevent suffocation and difficulty breathing before surgery, the lateral position should be taken, and the nasogastric tube should be taken at the same time. Nasal feeding should be carried out. The postoperative care is very important, mainly the extraction time of the nasogastric tube [28]. The general small mandible can be extended for 3-5 days. Eat. However, for the giant tongue with small jaw, due to the operation of the tongue, the activity of the tongue needs to be restored, and the upper and lower diameter of the tongue should be increased to eat. Therefore, the experimental infusion is started when the length is extended to 12 days. Do not take the stomach tube first, and wait until the child can eat before removing the stomach tube and starting to eat. If you do not pay attention to improving eating, it will cause growth and development disorders. In addition, the child must be in a lateral position within 12 days to improve breathing and reduce coughing in saliva. The mouth should be cleaned daily, especially under the incision [29]. Avoid coughing, inhalation of pneumonia, etc. during care. If necessary, inhalation may be carried out daily. Children with giant tongue disease may need a tongue reduction procedure to prevent interpersonal problems associated with large, prominent tongues and to reduce the negative effects of large tongues on teeth and jaw development.

\section{Summary}

Bibliometric analysis shows that the research on Beckwith Wiedemann syndrome nursing is in the exploratory period, but the overall research results are few. The researchers have formed initially but the professional and characteristic are insufficient. The research content covers a wide range, but the hotspots and characteristics Not prominent. Therefore, Beckwith Wiedemann syndrome nursing research is in its infancy, and it is necessary to focus on the improvement of research quality and the depth of research content based on ensuring the research atmosphere. The Beckwith Wiedemann syndrome nursing research is continuously embodied and featured, and Beckwith is promoted in the true sense. The development of Wiedemann syndrome care.

\section{References}

[1] Brioude F, Netchine I, Praz F, et al. Mutations of the Imprinted CDKN1C Gene as a Cause of the Overgrowth Beckwith-Wiedemann Syndrome: Clinical Spectrum and Functional Characterization [J]. Human mutation, 2015; 36 (9): 894-902.

[2] Tunster SJ, Van de Pette M, John RM. Fetal overgrowth in the Cdkn1c mouse model of Beckwith-Wiedemann syndrome [J]. Disease models \& mechanisms, 2011; 4 (6): 814-821.

[3] Poole RL, Leith DJ, Docherty LE, et al. Beckwith-Wiedemann syndrome caused by maternally inherited mutation of an OCT-binding motif in the IGF2/H19-imprinting control region, ICR1 [J]. European journal of human genetics: EJHG, 2012; 20 (2): $240-243$.

[4] Duquesnes N, Callot C, Jeannot P, et al. p57 (Kip2) knock-in mouse reveals CDK-independent contribution in the development of Beckwith-Wiedemann syndrome $[\mathrm{J}]$. The Journal of pathology, 2016; 239 (3): 250-261.

[5] Gripp KW, Robbins KM, Sheffield BS, et al. Paternal uniparental disomy $11 \mathrm{p} 15.5$ in the pancreatic nodule of an infant with Costello syndrome: Shared mechanism for hyperinsulinemic hypoglycemia in neonates with Costello and Beckwith-Wiedemann syndrome and somatic loss of heterozygosity in Costello syndrome driving clonal expansion [J]. American journal of medical genetics Part A, 2016; 170 (3): 559-564.

[6] Ohtsuka Y, Higashimoto K, Sasaki K, et al. Autosomal recessive cystinuria caused by genome-wide paternal uniparental isodisomy in a patient with Beckwith-Wiedemann syndrome [J]. Clinical genetics, 2015; 88 (3): 261-266. 
[7] Batra M, Valecha UK. Anesthetic management of tongue reduction in a case of Beckwith-Wiedemann syndrome [J]. Journal of anaesthesiology, clinical pharmacology, 2014; 30 (4): $562-564$.

[8] Tunster SJ, Van de Pette M, Creeth HDJ, et al. Fetal growth restriction in a genetic model of sporadic Beckwith-Wiedemann syndrome [J]. Disease models \& mechanisms, 2018; 11 (11).

[9] Singh VB, Sribenja S, Wilson KE, et al. Blocked transcription through KvDMR1 results in absence of methylation and gene silencing resembling Beckwith-Wiedemann syndrome [J]. Development (Cambridge, England), 2017; 144 (10): 1820-1830.

[10] Jacob KJ, Robinson WP, Lefebvre L. Beckwith-Wiedemann and Silver-Russell syndromes: opposite developmental imbalances in imprinted regulators of placental function and embryonic growth [J]. Clinical genetics, 2013; 84 (4): 326-334.

[11] White JC, Liu J, Nahar A. Simultaneous Presentation of Wilms Tumor and Immature Ovarian Teratoma in Beckwith-Wiedemann Syndrome [J]. Journal of pediatric hematology/oncology, 2018; 40 (1): e61-e63.

[12] Udayakumaran S, Onyia CU. Beckwith-Wiedemann syndrome and Chiari I malformation--a case-based review of central nervous system involvement in hemihypertrophy syndromes [J]. Child's nervous system: ChNS: official journal of the International Society for Pediatric Neurosurgery, 2015; 31 (5): 637-641.

[13] Knopp C, Rudnik-Schoneborn S, Zerres K, et al. Twenty-one years to the right diagnosis - clinical overlap of Simpson-Golabi-Behmel and Beckwith-Wiedemann syndrome [J]. American journal of medical genetics Part A, 2015; 167a (1): $151-155$.

[14] Van Lierde K, Galiwango G, Hodges A, et al. Impact of tongue reduction on overall speech intelligibility, articulation and oromyofunctional behavior in 4 children with Beckwith-Wiedemann syndrome [J]. Folia phoniatrica et logopaedica: official organ of the International Association of Logopedics and Phoniatrics (IALP), 2012; 64 (2): 55-63.

[15] Abi Habib W, Azzi S, Brioude F, et al. Extensive investigation of the IGF2/H19 imprinting control region reveals novel OCT4/SOX2 binding site defects associated with specific methylation patterns in Beckwith-Wiedemann syndrome [J]. Human molecular genetics, 2014; 23 (21): 5763-5773.

[16] Duffy KA, Cielo CM, Cohen JL, et al. Characterization of the Beckwith-Wiedemann spectrum: Diagnosis and management $[J]$. American journal of medical genetics Part C, Seminars in medical genetics, 2019.

[17] Wang Q, Geng Q, Zhou Q, et al. De novo paternal origin duplication of chromosome 11p15.5: report of two Chinese cases with Beckwith-Wiedemann syndrome [J]. Molecular cytogenetics, 2017; 10: 46.

[18] MacFarland SP, Duffy KA, Bhatti TR, et al. Diagnosis of Beckwith-Wiedemann syndrome in children presenting with Wilms tumor [J]. Pediatric blood \& cancer, 2018; 65 (10): e27296.

[19] Kalish JM, Deardorff MA. Tumor screening in Beckwith-Wiedemann syndrome-To screen or not to screen? [J]. American journal of medical genetics Part A, 2016; 170 (9): 2261-2264.

[20] Soussi-Zander C. Beckwith-Wiedemann Syndrome Revisited [J]. Human mutation, 2015; 36 (9): iii.

[21] Russo S, Calzari L, Mussa A, et al. A multi-method approach to the molecular diagnosis of overt and borderline $11 \mathrm{p} 15.5$ defects underlying Silver-Russell and Beckwith-Wiedemann syndromes [J]. Clinical epigenetics, 2016; 8: 23.

[22] Mussa A, Di Candia S, Russo S, et al. Recommendations of the Scientific Committee of the Italian Beckwith-Wiedemann Syndrome Association on the diagnosis, management and follow-up of the syndrome $[\mathrm{J}]$. European journal of medical genetics, 2016; 59 (1): 52-64.

[23] Brioude F, Hennekam R, Bliek J, et al. Revisiting Wilms tumour surveillance in Beckwith-Wiedemann syndrome with IC2 methylation loss, reply [J]. European journal of human genetics: EJHG, 2018; 26 (4): 471-472.

[24] Davlin AS, Clarkin CM, Kalish JM. Beckwith-Wiedemann Syndrome: Partnership in the Diagnostic Journey of a Rare Disorder [J]. Pediatrics, 2018; 141 (3).

[25] Cammarata-Scalisi F, Avendano A, Stock F, et al. Beckwith-Wiedemann syndrome: clinical and etiopathogenic aspects of a model genomic imprinting entity [J]. Archivos argentinos de pediatria, 2018; 116 (5): 368-373.

[26] Mussa A, Russo S, De Crescenzo A, et al. Prevalence of Beckwith-Wiedemann syndrome in North West of Italy [J]. American journal of medical genetics Part A, 2013; 161a (10): 2481-2486.

[27] Weksberg R, Shuman C, Beckwith JB. Beckwith-Wiedemann syndrome $[\mathrm{J}]$. European journal of human genetics: EJHG, 2010; 18 (1): 8-14.

[28] Aoki A, Shiozaki A, Sameshima A, et al. Beckwith-Wiedemann syndrome with placental chorangioma due to H19-differentially methylated region hypermethylation: a case report $[\mathrm{J}]$. The journal of obstetrics and gynaecology research, 2011; 37 (12): 1872-1876.

[29] Armes JE, McGown I, Williams M, et al. The placenta in Beckwith-Wiedemann syndrome: genotype-phenotype associations, excessive extravillous trophoblast and placental mesenchymal dysplasia [J]. Pathology, 2012; 44 (6): 519-527. 